\title{
Transient MHD boundary-layer slip-flow of heat and mass transfer over a stretching surface embedded in porous medium with waste discharge concentration and convective boundary conditions
}

\author{
Adetunji Adeniyan 1, *, Joshua Aanuoluwapo Adigun ${ }^{2}$ \\ ${ }^{1}$ Department of Mathematics, University of Lagos, Akoka, Yaba, Lagos State, Nigeria \\ ${ }^{2}$ Department of Physical Sciences, Bells University of Technology, Ota, Ogun State, Nigeria
}

Email addresses:

aadeniyan@unilag.edu.ng (A. Adeniyan), engraanuadigun@yahoo.com (J. A. Adigun)

To cite this article:

Adetunji Adeniyan, Joshua Aanuoluwapo Adigun. Transient MHD Boundary-Layer Slip-Flow of Heat and Mass Transfer Over a Stretching Surface Embedded in Porous Medium with Waste Discharge Concentration and Convective Boundary Conditions. Applied and Computational Mathematics. Vol. 3, No. 5, 2014, pp. 247-255. doi: 10.11648/j.acm.20140305.19

\begin{abstract}
The transient two-dimensional MHD boundary-layer stagnation point flow with heat and mass transfer in a saturated porous medium is presented here by taking into account the transient dispersion of a pollutant spewed by an external source in the presence of a uniform transverse magnetic field and stress (pressure) work. The laminar flow of viscous incompressible and electrically conducting fluid encompassing a convectively heated stationary permeable sheet is assumed to be described in terms of Darcian law. The nonlinear governing partial differential equations obtained are converted into ordinary differential equations by means of appropriate similarity transformations and consequently solved numerically using the forth order Runge-Kutta method with a shooting technique and depicted graphically for some pertinent values of the physical parameters embedded in the flow model. In addition, the skin-friction coefficient, the heat and pollution mass concentration rates are sorted out in tabular form, analyzed and discussed. We opine that findings of this present study will be found useful for environmental systems in pollution control and ventilation, and serve as complementary reference for researchers.
\end{abstract}

Keywords: Forced Convection, Permeable Sheet, Porous Medium, Pollutant Concentration, Magnetohydrodynamics, Convective Boundary Condition

\section{Introduction}

Influences of pollution on the environmental fluid flow cannot be over-emphasized due to their prevalent havocs wreaked on life, properties and economy. Much harm has been associated with the pollution of air and water environment. Air occupies about $99.9 \%$ of the universe, water resides a larger expanse of the earth. Discharge of some gaseous substances from industrial wastes, chimneys, ground and underwater vehicles, space crafts, gas turbines and a host of others have been attributed not only to the depletion of ozone layers but also unhealthy environment. Circumstances of oil spillage, discharge of toxic wastes and/ or other various aspects of contaminants into the seas, rivers, estuaries, wells/bore holes, etc are subject matters of these days. In real life situation, the presence of clean air or water is almost impossible. Many a researcher's attention has been attracted to investigate and divulge various ways of stemming the menaces associated with boundary layer flow of fluid in which pollutant concentration or advectiondispersion is significant. A score of authors such as Makinde and Chinyoka [1], Makinde et al. [2], Moitsheki and Makinde [3, 4], Chinyoka and Makinde [5], amongst many others investigated theoretically by constructing appropriate pollutant concentration source functions along with other fluid properties for their mathematical models for fluid flows in a channel or a pipe. Odor characteristics of engineering / industrial processes of paper, pulp and polymer sheets may constitute another source of pollutant concentration bearing in its effects to the manufactured end products as well as health hazard if not properly controlled. More recently Lakshminarayanachari et al. [6] demonstrated 
in their analysis the removal mechanisms through wet deposition, dry deposition and gravitational settling processes of the pollutants emitted from the urban area source. Shekhu and Sulochana [7] who considered the pollutants emitted from a time-dependent line source as a step function and used the Laplace transform method in their analysis. In the industrial processes after extrusion from the mills, during a roll-up for air-cooled paper and polymer sheets or water cooled polymer sheet, the uncontrolled industrially injected pollutants sometimes have consequential influences on the penultimate manufactured products, for example the discoloration effect as reported by Andersson et al.[8]), Abel et al.[9] and Mukhopadhyay [10], the knowledge of heat and flow fields near the plate is very essential for better understanding of the factors determining the quality of the final manufactured products. Utilizing the method of classical Lie symmetry analysis, Makinde et al.[2] obtained some invariant solutions for river pollution problem and nonlocal (potential) symmetry. Forced and/or free convective heat transfer in a saturated porous medium has been a topic of special interest in the most recent years. The research activities have received more serious attention, in this conceptual field due to its wider range of applications in various disciplines namely the pollutant dispersion in aquifers, agriculture, ventilation of rooms, enhanced crude oil extraction, glass blowing, wire and fiber tinning, annealing and coating, food production technology, nuclear waste repositories [see Nield and Bejan [11], Ingham and Pop [12], Pop and Ingham [13]), Ishak et al.[14], Olanrewaju et al.[15], Adeniyan and Adigun [16]. One common example of heat transfer is that demonstrated by vehicle radiator, in which the hot radiator fluid is cooled by the flow of air over the radiator surface.

Not much studies have been reported to date in the literature investigating the influence of pollutant dispersion on heat and mass transfer boundary layer flow over a stationary or a moving sheet in spite of the relevance of such flow in practice and the much needed consequential information on the abatement of pollutants. Therefore, the objective of this communication is to study the MHD heat and mass transfer stagnation point flow of an incompressible, viscous and electrically conducting fluid over a convectively heated horizontal plate in the presence of a transverse magnetic field, taking into cognizance the simultaneous influences of the stress (pressure) work and nonlinear pollutants (effluents) emitted from a source.

\section{Mathematical Formulation}

We consider a transient, two dimensional stagnation point flow of a viscous and incompressible fluid over a stationary horizontal surface embedded in a saturated porous medium as shown in Fig.1. The fluid is electrically conducting and exposed to a uniform magnetic field of strength $B_{0}$ applied normal to the plane surface, both the fluid and porous medium are in thermal equilibrium and the flow is laminar. The induced magnetic field and Hall current are neglected under the assumption of a small magnetic Reynolds number. Initially at time $\mathrm{t}=0$, a given pollutant is injected (spewed) into the flow regime from an external source which varies with its concentration according to the exponential law given by eq. 5 below, see Makinde and Chinyoka [1] in which $\mathrm{C}$ is the pollutant concentration. Far from the plate, the free stream velocity is of the form $u_{e}=a x$, where a $>0$ is a constant which has the unit of reciprocal of time, $\mathrm{t}^{-1}$ and $\mathrm{x}$ is the coordinate measured along the plate surface whereas the coordinate $\mathrm{y}$ is measured along the normal to the plate. At time $\mathrm{t}>0$, it is assumed that the surface beneath the plate is heated convectively by a hot fluid at temperature $T_{f}$ which provides a heat transfer coefficient $h_{f}$ and the wall temperature is $\mathrm{T}_{\mathrm{W}}$ consequential upon a heating process due to convection characterized by hot fluid wherein $T_{f}>T_{W}$. Also it is assumed that the local mass flux velocity $v_{w}$ is perpendicular to the plate such that its positive values are those of mass withdrawal or suction while negative values are those of mass blowing or injection. Taking all the foregoing assumptions into consideration, the governing equations for the boundary-layer flow of the fluid are as follows:

$$
\begin{gathered}
\frac{\partial u}{\partial x}+\frac{\partial \mathrm{v}}{\partial y}=0 \\
\frac{\partial \mathrm{u}}{\partial \mathrm{t}}+u \frac{\partial u}{\partial x}+\mathrm{v} \frac{\partial u}{\partial y}=\frac{\partial u_{e}}{\partial \mathrm{t}}+\mathcal{U}_{e} \frac{d u_{e}}{d x}+\gamma \frac{\partial^{2} u}{\partial y^{2}}-\frac{\sigma B_{0}^{2}}{\rho}\left(u-\mathcal{U}_{e}\right)- \\
\frac{\gamma}{K_{P}}\left(u-U_{e}\right)=0 \\
\frac{\partial \mathrm{T}}{\partial \mathrm{t}}+u \frac{\partial T}{\partial x}+\mathrm{v} \frac{\partial T}{\partial y}=\alpha \frac{\partial^{2} u}{\partial y^{2}}+\frac{1}{c_{P}} u_{e} \frac{d u_{e}}{d x}\left(u_{e}-u\right)=0 \\
\frac{\partial \mathrm{C}}{\partial \mathrm{t}}+u \frac{\partial C}{\partial x}+\mathrm{v} \frac{\partial C}{\partial y}=D_{m} \frac{\partial^{2} C}{\partial y^{2}}+S(c)
\end{gathered}
$$

Where the external pollutant concentration, Makinde and Chinyoka [1], is posited as

$$
S(c)=Q \exp \left(c-c_{\infty}\right) \phi(\eta)
$$

and Q is the pollutant source strength.

The corresponding boundary conditions are:

For $t \geq 0$

$$
\begin{aligned}
& u(t, x, 0)=\beta \frac{\partial u}{\partial y}(t, x, 0), \mathrm{v}(t, x, 0)=\mathrm{v}_{\mathrm{w}}, \\
& -k \frac{\partial T}{\partial y}(t, x, 0)=h_{f}\left(T_{f}-T(t, x, 0)\right) \\
& \left.\begin{array}{c}
u(t, x, \infty)=u_{e}=a x, T(t, x, \infty)=T_{\infty}, \\
C(t, x, \infty)=C_{\infty}
\end{array}\right\}
\end{aligned}
$$

where the fluid quantities $\mathrm{T}, T_{\infty}, C_{w}, C_{\infty}, \mathrm{k}, h_{f}, \beta$ are the temperature, free stream temperature,

pollutant concentration at the wall surface, free stream pollutant concentration, thermal

conductivity, convective heat transfer coefficient and slip length respectively as presented in 6 .

Following Makinde [17], it is convenient to use the following similarity transformations: 


$$
\left.\begin{array}{cc}
\eta=y \sqrt{\frac{a}{\gamma(1-\lambda t)}}, & \psi(x, y)=x \sqrt{\frac{a \gamma}{1-\lambda t}} f(\eta), \\
\theta(\eta)=\frac{T-T_{\infty}}{T_{f}-T_{\infty}}, & \varphi(\eta)=\frac{c-C_{\infty}}{C_{w}-C_{\infty}} \\
\text { and } & F_{W}=-\mathrm{v}_{w} \sqrt{\frac{1-\lambda t}{a \gamma}}
\end{array}\right\}
$$

As presented in $7, \psi, f(\eta), \theta(\eta), \varphi(\eta), F_{W}$ are respectively dimensionless transverse distance, dimensional and dimensionless stream functions, dimensionless fluid temperature, pollutant concentration and suction velocity or fluid withdrawal parameter.

Eq. 1 is identically satisfied and eqs. $2-4$ transform to become

$$
\begin{gathered}
f^{\prime \prime \prime}+f f^{\prime \prime}-\left(f^{\prime}\right)^{2}-A\left(f^{\prime}+\frac{1}{2} \eta f^{\prime \prime}-1\right)-[H a+D a]\left(f^{\prime}-1\right)+1=0 \\
\frac{1}{P r} \theta^{\prime \prime}+E c\left(1-f^{\prime}\right)+f \theta^{\prime}-\frac{1}{2} A \eta \theta^{\prime}=0 \\
\frac{1}{S c} \phi^{\prime \prime}+f \phi^{\prime}+\varsigma \mathrm{e}^{\mathrm{n} \phi}-\frac{1}{2} A \eta \phi^{\prime}=0
\end{gathered}
$$

The transformed boundary conditions also become

$$
\left.\begin{array}{c}
f^{\prime}(0)=\delta f^{\prime \prime}(0), f(0)=F_{w}, \\
-\theta^{\prime}(0)=B_{i}(1-\theta(0)), \phi(0)=1, \\
f(\infty)=1, \phi(\infty)=\theta(\infty)=0
\end{array}\right\}
$$

where the prime signifies the derivative with respect to $\eta$. The dimensionless parameters:

$$
\left.\begin{array}{c}
S c=\frac{\gamma}{D_{m}}, \mathrm{H} a=\frac{\sigma B_{0}{ }^{2}}{\rho a}(1-\lambda t), \operatorname{Pr}=\frac{\gamma}{\alpha}, \\
B i=\frac{h_{f}}{k} \sqrt{\frac{\gamma(1-\lambda t)}{\alpha}}, D a=\frac{\gamma}{k_{p}}(1-\lambda t), \\
E c=\frac{u_{e}^{2}}{C_{p}\left(T_{f}-T_{\infty}\right)}, A=\frac{\lambda}{a}, \varsigma=\frac{Q(1-\lambda t)}{a\left(C_{w}-C_{\infty}\right)} \\
n=b\left(C_{w}-C_{\infty}\right), \delta=\beta \sqrt{\frac{a}{\gamma(1-\lambda t)}}
\end{array}\right\}
$$

are respectively the Schmidt number, the local Hartman number, the Prandtl number, the local Biot number, the local Darcy number, the Eckert number, the unsteadiness parameter, the local pollutant external source parameter, the local pollutant external source variation parameter and slip parameter wherein $b$ has the unit of reciprocal of pollutant concentration as presented in 12. There is need to proffer solutions to the obtained similarity eqs. 8-11, a set of coupled highly nonlinear equations whose solutions are not exactly available.

\section{Numerical Procedure}

The set of equations $8-10$ under the boundary conditions 11 has been numerically solved using a shooting algorithm with a Runge-Kutta fourth order scheme. For full descriptions, see Bhattacharyya et al.[18] and Ibrahim and Makinde[19]. Firstly, it is pertinent to reduce the boundary value problem (BVP) into a set of first order initial value problems (IVP) and assuming a suitable far field dimensionless distance $\eta_{\max }$ corresponding to $\eta=\infty$ along with some unspecified parameters. Computations have been done by the Maple program and a step size employed guarantees accuracy at least to the fifth decimal place as criterion sufficient for convergence. The maximum value of the dimensionless distance made use of is $\eta_{\max }=3$ in all cases for which the unknown boundary conditions do not change to successful loop with error less than $10^{-7}$. From the process of numerical computations, the plate Surface temperature $\theta(0)$, the Skin-friction coefficient, Nusselt and Sherwood numbers which are respectively proportional to $f^{\prime \prime}(0),-\theta^{\prime}(0)$ and $-\phi^{\prime}(0)$ are also sorted out for their numerical values and presented in tabular form. The accuracy of this numerical method was validated by direct comparison with the numerical results reported by Makinde [17] and Dulal and Hiremath [20] for this particular investigation when $D a=0$ and in the absence of pollution. And a perfect agreement is observed, as demonstrated in Tables 1 and 2 below. It is worthy to emphasize that eq. 10 connotes ill expression when $\mathrm{Sc}=0$.

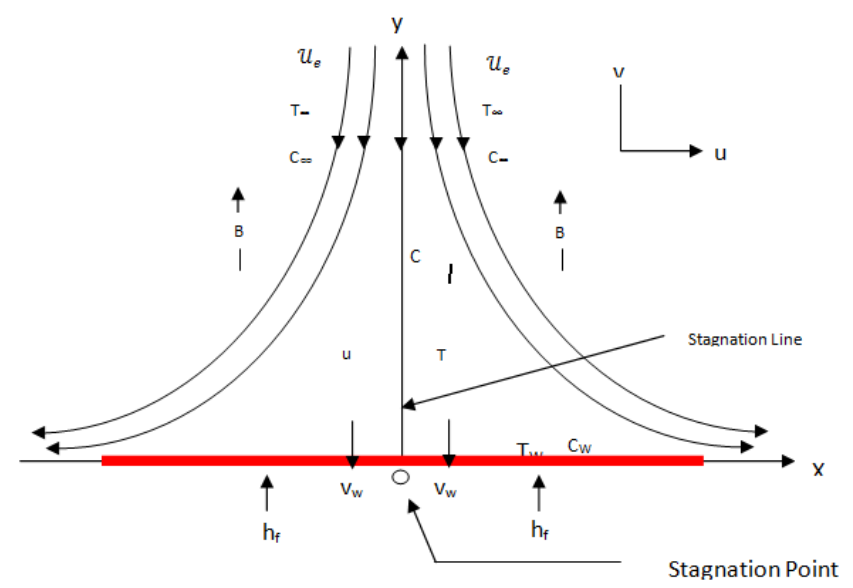

Fig 1. Physical model and coordinate system.

Also, we conducted a direct comparison with the numerical results reported by Dulal and Hiremath [20] for the unsteady boundary layer flow over a moving plate in the absence of heat and mass transfer modeled as

$$
\begin{gathered}
f^{\prime \prime \prime}+f f^{\prime \prime}-\left(f^{\prime}\right)^{2}-A\left(f^{\prime}+\frac{1}{2} \eta f^{\prime \prime}\right)=0, f(0)=0, \\
f^{\prime}(0)=1, f^{\prime}(\infty)=0
\end{gathered}
$$

The results in Table 2 below show an almost perfect agreement with those of Dulal and Hiremath [20]. 
250 Adetunji Adeniyan and Joshua Aanuoluwapo Adigun: Transient MHD Boundary-Layer Slip-Flow of Heat and Mass Transfer Over a Stretching Surface Embedded in Porous Medium with Waste Discharge Concentration and Convective Boundary Conditions

Table 1. Computations showing comparison with Makinde [17] for $F_{w}=E c=D a=\varsigma=n=0$, using his governing flow parametric values.

\begin{tabular}{ccccccccccc}
\hline $\boldsymbol{P r}$ & $\boldsymbol{A}$ & $\boldsymbol{B i}$ & $\boldsymbol{H a}$ & $\boldsymbol{\delta}$ & $\begin{array}{c}\boldsymbol{f}^{\prime \prime}(\mathbf{0}) \\
\text { Makinde [17] }\end{array}$ & $\begin{array}{c}-\boldsymbol{\theta}^{\prime}(\mathbf{0}) \\
\text { Makinde [17] }\end{array}$ & $\begin{array}{c}\boldsymbol{\theta}(\mathbf{0}) \\
\text { Makinde [17] }\end{array}$ & $\begin{array}{c}\boldsymbol{f}^{\prime \prime}(\mathbf{0}) \\
\text { Present }\end{array}$ & $\begin{array}{c}-\boldsymbol{\theta}^{\prime}(\mathbf{0}) \\
\text { Present }\end{array}$ & $\begin{array}{c}\boldsymbol{\theta}(\mathbf{0}) \\
\text { Present }\end{array}$ \\
\hline 0.72 & 0.1 & 0.1 & 0.1 & 0.1 & 1.186380878 & 0.08358870 & 0.16411293 & 1.18650347 & 0.08401833 & 0.15981669 \\
3.00 & 0.1 & 0.1 & 0.1 & 0.1 & 1.186380878 & 0.09000268 & 0.09997318 & 1.18650347 & 0.09000342 & 0.09996575 \\
0.72 & 0.1 & 0.5 & 0.1 & 0.1 & 1.186380878 & 0.25231253 & 0.49537492 & 1.18650347 & 0.25626801 & 0.48746396 \\
0.72 & 0.1 & 0.1 & 0.1 & 3.0 & 0.275659634 & 0.08635366 & 0.13646331 & 0.27566170 & 0.08635340 & 0.13465944 \\
\hline
\end{tabular}

Table 2. Computations showing comparison of the skin friction $f^{\prime \prime}(0)$ with Dulal and Hiremath [20] for $\operatorname{Pr}=S c=B i=H a=F_{w}=E c=D a=\varsigma=$ $n=\delta=0$, using their governing flow parametric values

\begin{tabular}{|c|c|c|}
\hline $\boldsymbol{A}$ & $f^{\prime \prime}(0)$ D-H [20] & $f^{\prime \prime}(0)$ Present \\
\hline 0.5 & -1.167221 & -1.16721482 \\
\hline 1.0 & -1.320540 & -1.32053488 \\
\hline 1.5 & -1.459687 & -1.45968356 \\
\hline 2.0 & -1.587403 & -1.58739857 \\
\hline
\end{tabular}

\section{Results and Discussion}

Numerical calculations have been carried out for different values of the control thermophysical parameters in the flow regime. The values of Schmidt number $(S c)$ for various types of fluid were chosen; water vapor $(S c=0.62)$, ammonia $(S c=0.78)$ and Propy Benzene $(S c=2.62)$ at temperature $25^{\circ} \mathrm{C}$ and one athmospheric pressure. In all computations, we desire the variation of $f, \theta$ and $\phi$ versus $\eta$ for the velocity, temperature and pollutant diffusion boundary layers. Table 3 shows the computations for the influence of the embedded flow parameters on the skin-friction coefficient and temperature together with heat and mass transfer rates at the moving plate surface. It is quite interesting to note that the temperature gradient $\theta^{\prime}(0)$ is negative for all parameters considered in this study. This simply implies that the heat flow is from the hot fluid at the lower surface of the plate to the cold fluid on the upper surface of the plate. It was observed that the skin-friction coefficient was increased by increasing values of Hartman or magnetic field, permeability and unsteadiness parameters. However, the skin friction coefficient was seen to decrease as more fluid was withdrawn from the flow system and with increasing values of the Navier-slip parameter. All other thermophysical parameters had no effect on it. Furthermore, the plate temperature is positively influenced by ascending values of Prandtl number, Hartman number, Eckert number, fluid withdrawal, permeability and unsteadiness parameters. The plate temperature is decreased by increasing values of Biot number and the Navier slip parameter while pollutant source parameters and Schmidt number had no influence on it. It's also of key note that the rate of heat transfer was increased by increasing values of all the thermophysical parameters except for the unsteadiness parameter which decreased it and other three (Schmidt number, local pollutant external source parameter and the local pollutant external source variation parameter) which had neither a decreasing nor an increasing effect on it. The external pollutant source parameter, Navier slip parameter and Schmidt number all increased the rate at which fluid mass was transferred from the plate surface to the far stream within the boundary layer but the Hartman number, fluid withdrawal, unsteadiness and permeability parameters decreased it.

\subsection{Velocity Profiles}

The illustration of the velocity profiles with respect to the transverse distance is displayed in Figs.2 to 5. Generally, the flow velocity is lowest at the plate surface and increases gradually to its free stream value of 1 , thereby satisfying the far field boundary condition. In Fig.2, an increase was observed in the flow velocity with an increase in the flow unsteadiness. Fig. 3 shows that there is an increase in the fluid velocity as there is a continual fluid withdrawal from the flow system. Furthermore, Fig.4 shows that there is a very slight increase in the fluid velocity at regions close to the plate with accompanied flow reversal far from the plate. As expected, an increased magnetic field which represents a damping effect on the velocity by creating a drag force which opposes fluid motion led to an imminent decrease in flow velocity. Lastly, Fig.5 shows an increase in the flow velocity as the Navier slip parameter is being increased.

Table 3. Computations showing the friction coefficient $\left(f^{\prime \prime}(0)\right)$, surface temperature $(\theta(0))$, Nusselt number $\left(-\theta^{\prime}(0)\right)$ and Sherwood number $\left(-\phi^{\prime}(0)\right.$ for various values of basic flow parameters.

\begin{tabular}{|c|c|c|c|c|c|c|c|c|c|c|c|c|c|c|}
\hline$P r$ & $S c$ & $B i$ & $\mathrm{Ha}$ & $E \boldsymbol{C}$ & $D a$ & $A$ & $F_{w}$ & $\delta$ & $\varsigma$ & $n$ & $f^{\prime \prime}(0)$ & $\boldsymbol{\theta}(\mathbf{0})$ & $-\boldsymbol{\theta}^{\prime}(\mathbf{0})$ & $-\boldsymbol{\phi}^{\prime}(\mathbf{0})$ \\
\hline 0.72 & 0.24 & 0.1 & 0.1 & 0.5 & 0.5 & 1.0 & 1.0 & 0.1 & 0.5 & 0.1 & 0.755587 & 13.02503 & 1.202503 & 0.042465 \\
\hline 1.00 & 0.24 & 0.1 & 0.1 & 0.5 & 0.5 & 1.0 & 1.0 & 0.1 & 0.5 & 0.1 & 0.755587 & 16.38371 & 1.538371 & 0.042465 \\
\hline 2.00 & 0.24 & 0.1 & 0.1 & 0.5 & 0.5 & 1.0 & 1.0 & 0.1 & 0.5 & 0.1 & 0.755587 & 21.52032 & 2.052032 & 0.042465 \\
\hline 0.72 & 0.62 & 0.1 & 0.1 & 0.5 & 0.5 & 1.0 & 1.0 & 0.1 & 0.5 & 0.1 & 0.755587 & 13.02503 & 1.202503 & 0.623774 \\
\hline 0.72 & 0.78 & 0.1 & 0.1 & 0.5 & 0.5 & 1.0 & 1.0 & 0.1 & 0.5 & 0.1 & 0.755587 & 13.02503 & 1.202503 & 0.790732 \\
\hline 0.72 & 2.62 & 0.1 & 0.1 & 0.5 & 0.5 & 1.0 & 1.0 & 0.1 & 0.5 & 0.1 & 0.755587 & 13.02503 & 1.202503 & 0.982070 \\
\hline 0.72 & 0.24 & 0.5 & 0.1 & 0.5 & 0.5 & 1.0 & 1.0 & 0.1 & 0.5 & 0.1 & 0.755587 & 3.638351 & 1.319175 & 0.042465 \\
\hline 0.72 & 0.24 & 0.1 & 0.5 & 0.5 & 0.5 & 1.0 & 1.0 & 0.1 & 0.5 & 0.1 & 0.953702 & 13.68118 & 1.268118 & 0.041355 \\
\hline 0.72 & 0.24 & 0.1 & 1.0 & 0.5 & 0.5 & 1.0 & 1.0 & 0.1 & 0.5 & 0.1 & 1.161944 & 14.54283 & 1.354283 & 0.040285 \\
\hline 0.72 & 0.24 & 0.1 & 1.5 & 0.5 & 0.5 & 1.0 & 1.0 & 0.1 & 0.5 & 0.1 & 1.337703 & 15.44604 & 1.444604 & 0.039507 \\
\hline 0.72 & 0.24 & 0.1 & 0.1 & 1.0 & 0.5 & 1.0 & 1.0 & 0.1 & 0.5 & 0.1 & 0.755587 & 25.16062 & 2.416062 & 0.042465 \\
\hline
\end{tabular}




\begin{tabular}{|c|c|c|c|c|c|c|c|c|c|c|c|c|c|c|}
\hline $\mathrm{Pr}$ & $S c$ & $B i$ & $H a$ & $E c$ & $D a$ & $A$ & $F_{w}$ & $\delta$ & $\varsigma$ & $n$ & $f^{\prime \prime}(0)$ & $\theta(\mathbf{0})$ & $-\boldsymbol{\theta}^{\prime}(\mathbf{0})$ & $-\phi^{\prime}(\mathbf{0})$ \\
\hline 0.72 & 0.24 & 0.1 & 0.1 & 2.0 & 0.5 & 1.0 & 1.0 & 0.1 & 0.5 & 0.1 & 0.755587 & 49.43181 & 4.827356 & 0.042465 \\
\hline 0.72 & 0.24 & 0.1 & 0.1 & 0.5 & 1.0 & 1.0 & 1.0 & 0.1 & 0.5 & 0.1 & 0.998462 & 13.84986 & 1.284986 & 0.041112 \\
\hline 0.72 & 0.24 & 0.1 & 0.1 & 0.5 & 2.0 & 1.0 & 1.0 & 0.1 & 0.5 & 0.1 & 1.369715 & 15.63102 & 1.463102 & 0.039376 \\
\hline 0.72 & 0.24 & 0.1 & 0.1 & 0.5 & 0.5 & 2.0 & 1.0 & 0.1 & 0.5 & 0.1 & 1.665008 & 0.299525 & 0.070047 & 0.512508 \\
\hline 0.72 & 0.24 & 0.1 & 0.1 & 0.5 & 0.5 & 3.0 & 1.0 & 0.1 & 0.5 & 0.1 & 1.792620 & 0.335895 & 0.066410 & 0.482316 \\
\hline 0.72 & 0.24 & 0.1 & 0.1 & 0.5 & 0.5 & 1.0 & -1.0 & 0.1 & 0.5 & 0.1 & 0.915099 & 5.897890 & 0.489789 & 0.075596 \\
\hline 0.72 & 0.24 & 0.1 & 0.1 & 0.5 & 0.5 & 1.0 & -0.5 & 0.1 & 0.5 & 0.1 & 0.894442 & 8.566498 & 0.756649 & 0.062346 \\
\hline 0.72 & 0.24 & 0.1 & 0.1 & 0.5 & 0.5 & 1.0 & 0.0 & 0.1 & 0.5 & 0.1 & 0.789544 & 12.21324 & 1.121324 & 0.046068 \\
\hline 0.72 & 0.24 & 0.1 & 0.1 & 0.5 & 0.5 & 1.0 & 1.0 & 0.1 & 0.5 & 0.1 & 0.197195 & 19.61298 & 1.861298 & 0.005209 \\
\hline 0.72 & 0.24 & 0.1 & 0.1 & 0.5 & 0.5 & 1.0 & 1.0 & 1.0 & 0.5 & 0.1 & 0.658320 & 0.244927 & 0.075507 & 0.197884 \\
\hline 0.72 & 0.24 & 0.1 & 0.1 & 0.5 & 0.5 & 1.0 & 1.0 & 2.0 & 0.5 & 0.1 & 0.398525 & 0.204480 & 0.079551 & 0.203901 \\
\hline 0.72 & 0.24 & 0.1 & 0.1 & 0.5 & 0.5 & 1.0 & 1.0 & 0.1 & 0.6 & 0.1 & 0.755587 & 13.02503 & 1.202503 & 0.088833 \\
\hline 0.72 & 0.24 & 0.1 & 0.1 & 0.5 & 0.5 & 1.0 & 1.0 & 0.1 & 0.8 & 0.1 & 0.755587 & 13.02503 & 1.202503 & 0.182353 \\
\hline 0.72 & 0.24 & 0.1 & 0.1 & 0.5 & 0.5 & 1.0 & 1.0 & 0.1 & 0.01 & 0.0 & 0.755587 & 13.02503 & 1.202503 & 0.022156 \\
\hline 0.72 & 0.24 & 0.1 & 0.1 & 0.5 & 0.5 & 1.0 & 1.0 & 0.1 & 0.01 & 0.1 & 0.755587 & 13.02503 & 1.202503 & 0.042465 \\
\hline 0.72 & 0.24 & 0.1 & 0.1 & 0.5 & 0.5 & 1.0 & 1.0 & 0.1 & 0.01 & 0.5 & 0.755587 & 13.02503 & 1.202503 & 0.156980 \\
\hline
\end{tabular}

\subsection{Temperature Profiles}

The effects of various combined thermophysical parameters on the flow temperature are illustrated in Figs.6 to 12. In Figs. 6 to 9 and 11, we observed an increase in the wall temperature as the Prandtl number, unsteadiness parameter, Eckert number, Hartman number and permeability parameter are increased. Figs. 10 and 12 revealed an increase in the temperature profile when fluid withdrawal parameter and Biot number are increased respectively. It is observed that for all the thermophysical parameters discussed, the temperature varies both at the plate and within the boundary layer but comes to a zero value at the far stream, which satisfies the boundary condition. In fig. 9, the dimensionless fluid temperature decreases in values as more fluid is withdrawn from the flow regime, thus the thermal boundary layer thickness diminishes.

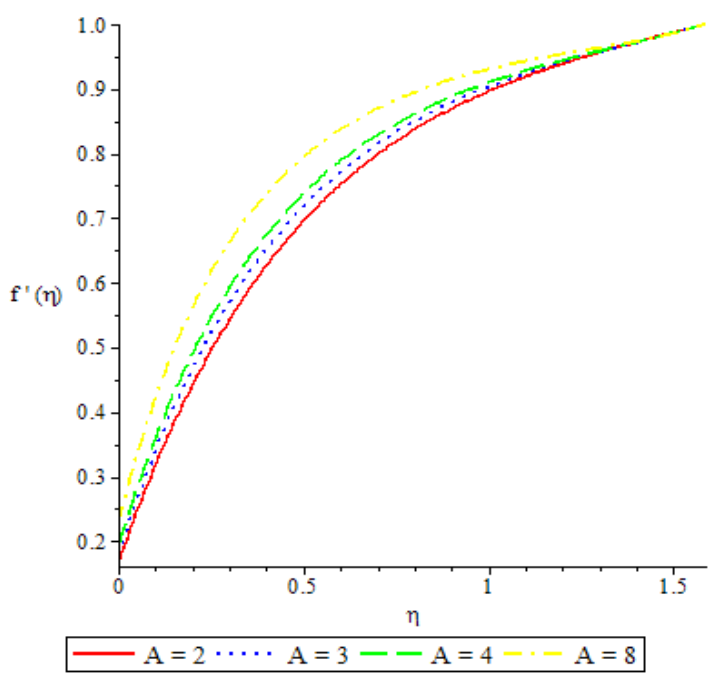

Figure 2. Velocity profiles for $\operatorname{Pr}=0.72, S c=0.24, E c=0.5, D a=$ $0.1, \varsigma=0.5$ and $n=\delta=B i=H a=F_{w}==0.1$.

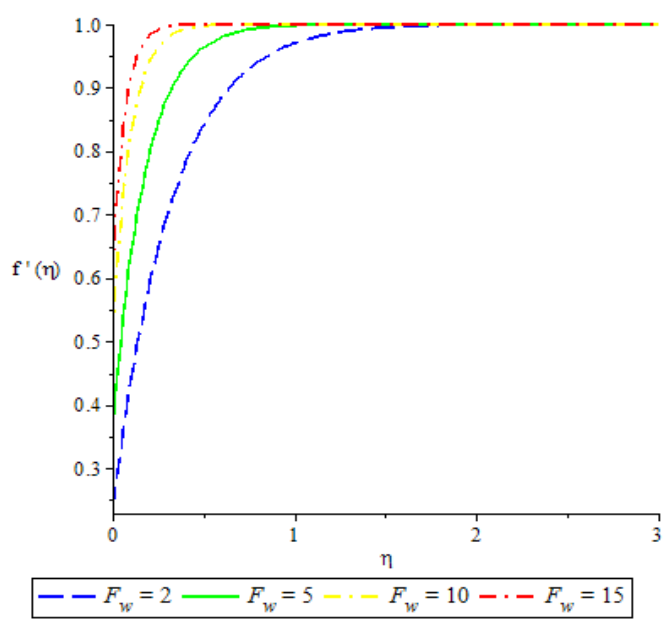

Figure 3. Velocity profiles for $\operatorname{Pr}=0.72, S c=0.24, E c=0.5, D a=$ $0.1, A=1.0, \varsigma=0.5$ and $n=\delta=B i=H a=0.1$.

\subsection{Concentration Profiles}

Figs. 13 to 17 show the concentration profiles. The boundary conditions are clearly satisfied as it was seen that the pollutant concentration had a value of 1 at the plate and moved to a minimum value of zero. Figs. 13 and 14 show a significant decrease in the concentration as there is more fluid withdrawal in the system and an increase in the local pollutant external source variation parameter. In Fig. 14, it was seen that as the level of external pollutant which infiltrated the flow system rose higher, there was a concurrent increase in the pollutant concentration. Fig. 15 described the influence of Schmidt number on pollutant concentration and it was observed that an increase in Schmidt number led to its increase within the boundary layer due to the effect of pollutant molecular diffusivity. Lastly, Figs. 16 and 17 described the influence of the local pollutant external source parameter and unsteadiness parameter on pollutant concentration. It was a similar observation that an increase in these thermophysical parameters all led to an increase in the pollutant concentration 
252 Adetunji Adeniyan and Joshua Aanuoluwapo Adigun: Transient MHD Boundary-Layer Slip-Flow of Heat and Mass Transfer Over a Stretching Surface Embedded in Porous Medium with Waste Discharge Concentration and Convective Boundary Conditions

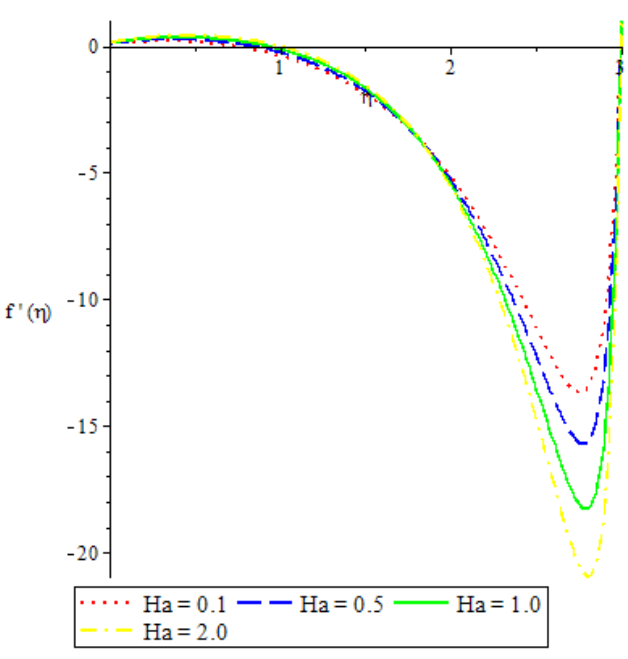

Figure 4. Velocity profiles for $\operatorname{Pr}=0.72, \delta=0.1, S c=0.24, E c=$ $0.5, A=1.0, \varsigma=0.5$ and $n=D a=B i=F_{w}=0.1$.

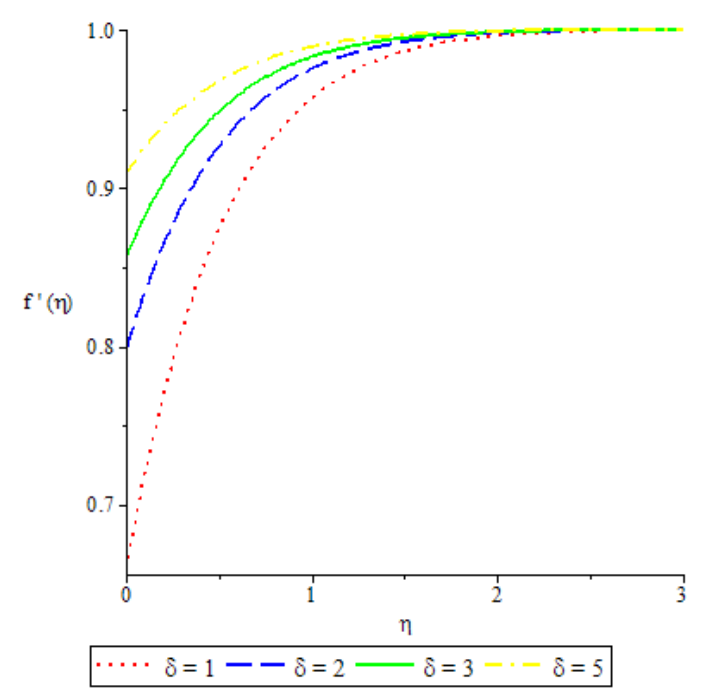

Figure 5. Velocity profiles for $\operatorname{Pr}=0.72, S c=0.24, E c=0.5, A=$ $1.0, \varsigma=0.5$ and $H a=B i=n=D a=F_{w}=0.1$.

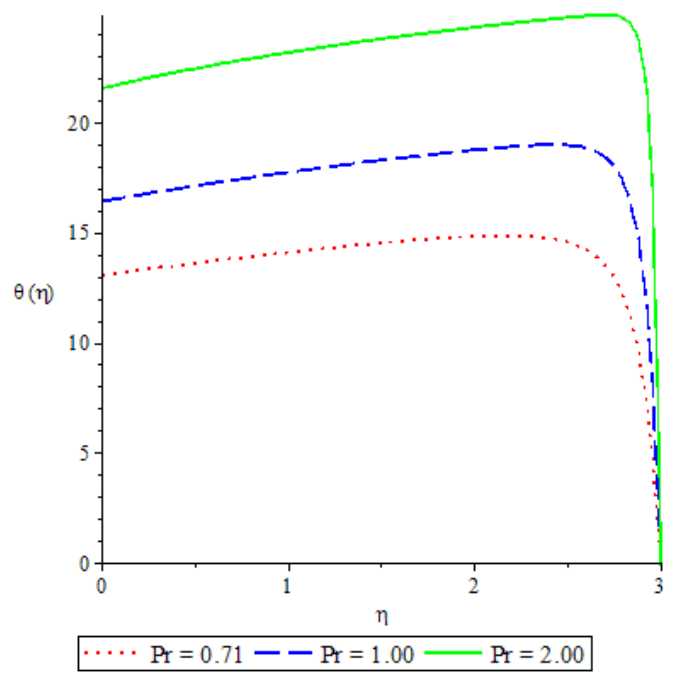

Figure 6. Temperature profiles for $S c=0.24, E c=0.5, A=1.0, \varsigma=$ 0.5 and $D a=n=B i=\delta=H a=F_{w}=0.1$

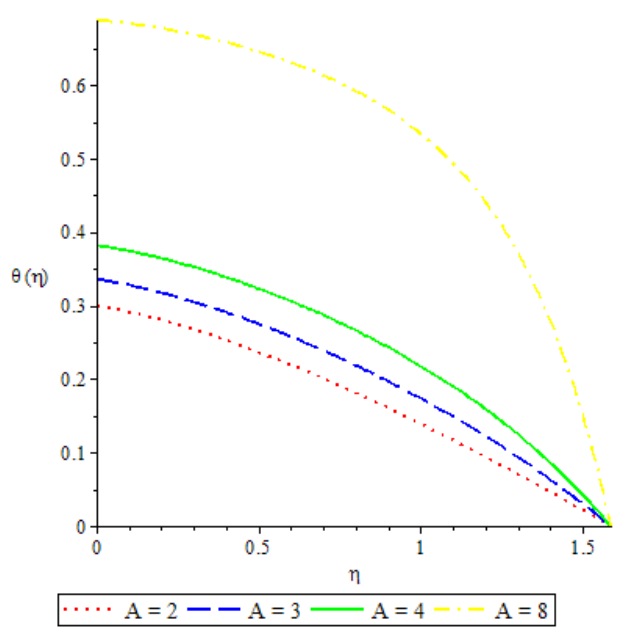

Figure 7. Temperature profiles for $\operatorname{Pr}=0.72, S c=0.24, E c=0.5, \varsigma=$ 0.5 and $D a=\delta=H a=F_{w}=n=0.1$.

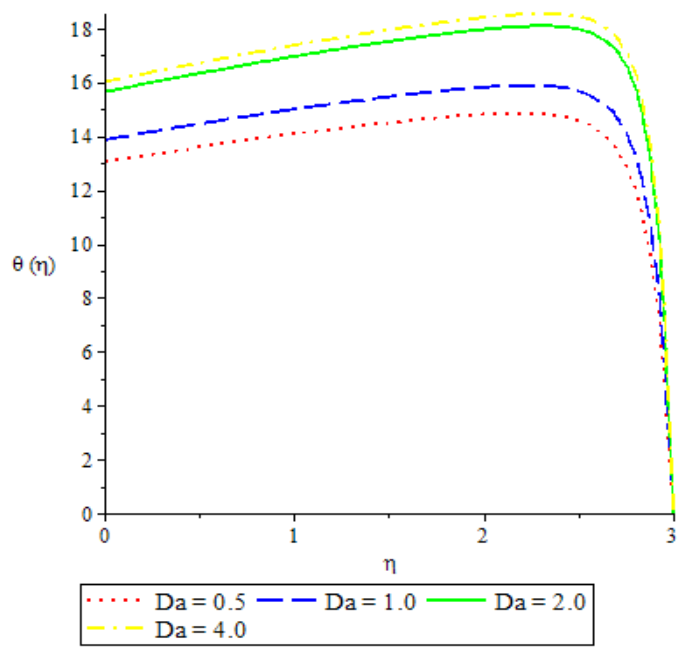

Figure 8. Temperature profiles for $\operatorname{Pr}=0.72, S c=0.24, E c=0.5,, A=$ $1.0, \varsigma=0.5$ and $B i=\delta=H a=F_{w}=n=0.1$.

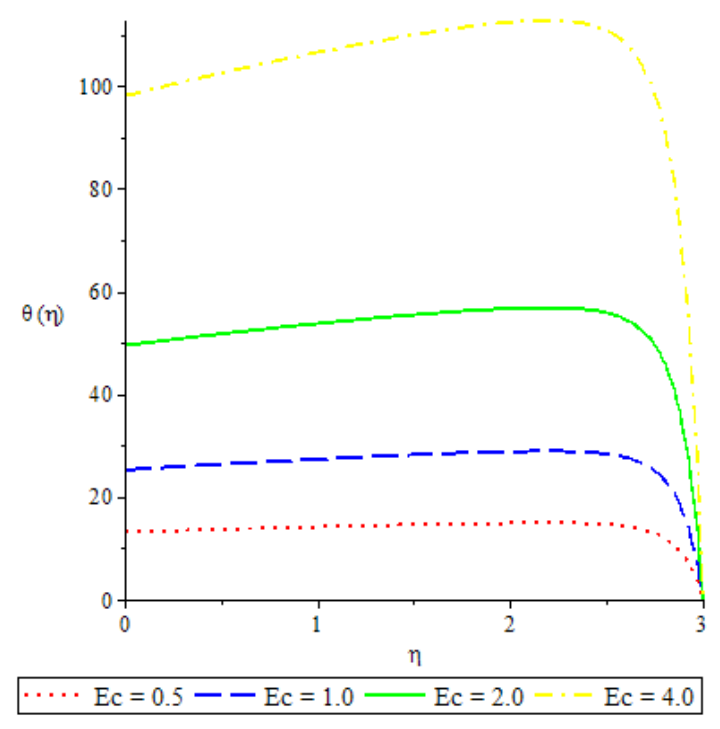

Figure 9. Temperature profiles for $\operatorname{Pr}=0.72, S c=0.24, A=1.0, \varsigma=$ 0.5 and $B i=\delta=H a=F_{w}=D a=n=0.1$. 


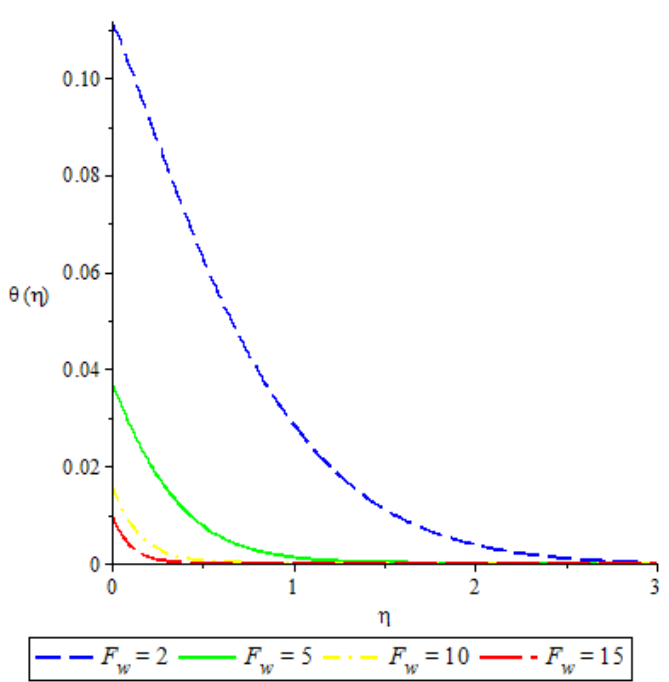

Figure 10. Temperature profiles for $\operatorname{Pr}=0.72, S c=0.24, E c=0.5, A=$ $1.0, \varsigma=0.5$ and $n=D a=B i=\delta=H a=0.1$.

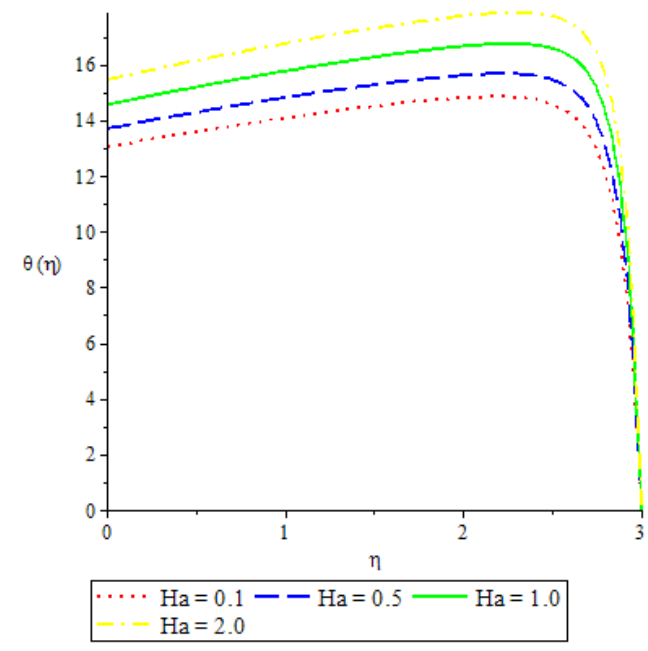

Figure 11. Temperature profiles for $\operatorname{Pr}=0.72, S c=0.24, E c=0.5, A=$ 1.0, $\varsigma=0.5$ and $B i=\delta=F_{w}=D a=n=0.1$.

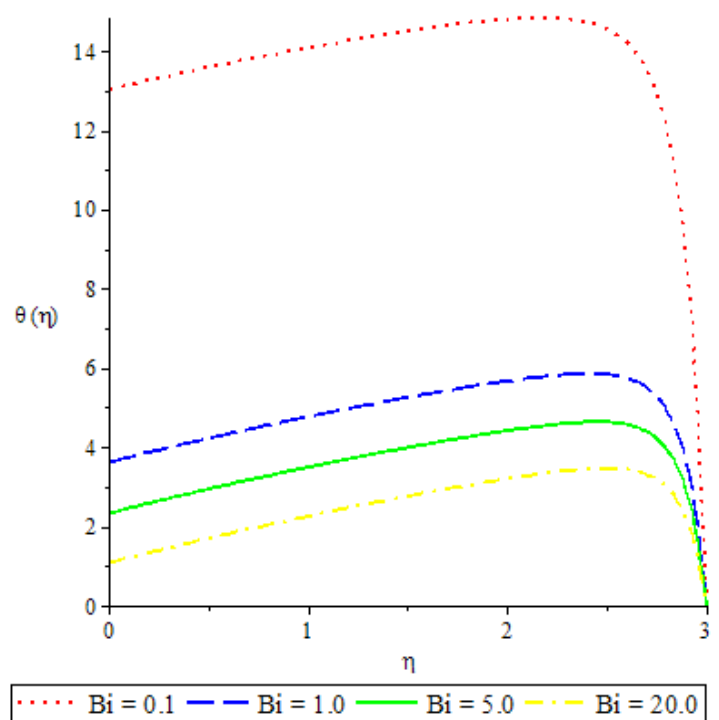

Figure 12. Temperature profiles for $\operatorname{Pr}=0.72, S c=0.24, E c=0.5, A=$ 1.0, $\varsigma=0.5$ and $H a=D a=\delta=F_{w}=n=0.1$

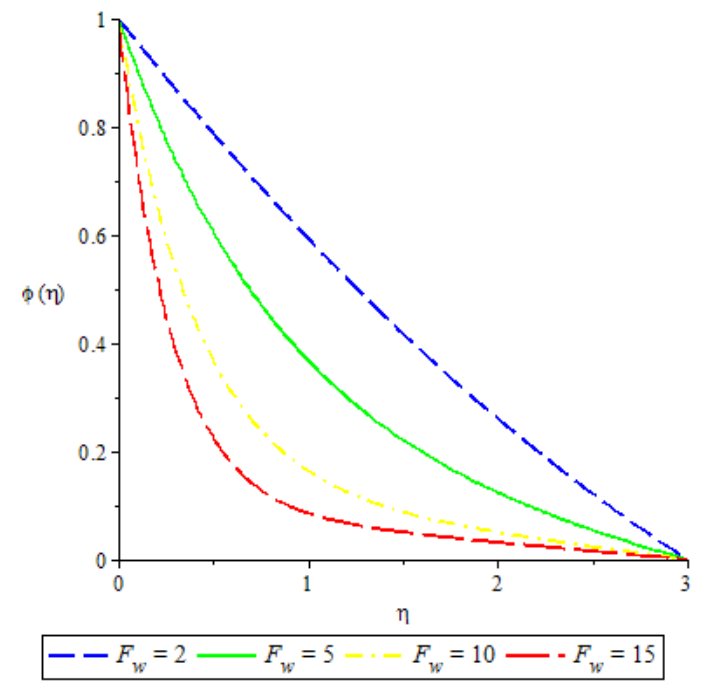

Figure 13. Concentration profiles for $\operatorname{Pr}=0.72, S c=0.24, E c=0.5, A=$ $1.0, \varsigma=0.5$ and $n=\delta=B i=H a=D a=0.1$.

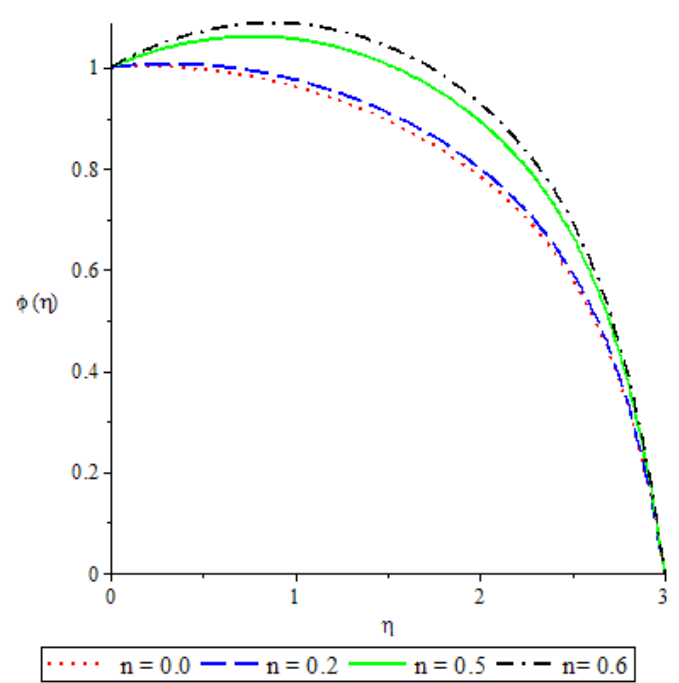

Figure 14. Concentration profiles for $\operatorname{Pr}=0.72, S c=0.24, E c=0.5, A=$ $1.0, \varsigma=0.5$ and $\delta=D a=B i=H a=F_{w}=0.1$

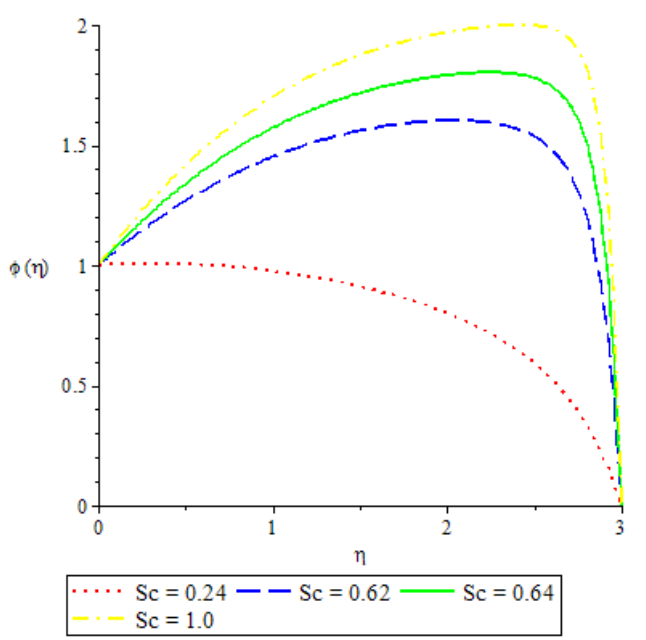

Figure 15. Concentration profiles for $\operatorname{Pr}=0.72, E c=0.5, A=1.0, \varsigma=$ 0.5 and $B i=H a=\delta=D a=F_{w}=n=0.1$ 


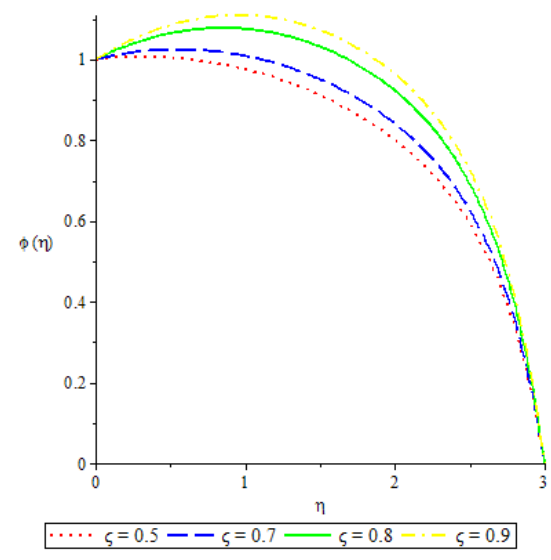

Figure 16. Concentration profiles for $\operatorname{Pr}=0.72, S c=0.24, E c=0.5, A=$ 1.0 and $n=B i=\delta=H a=F_{w}=D a=0.1$.

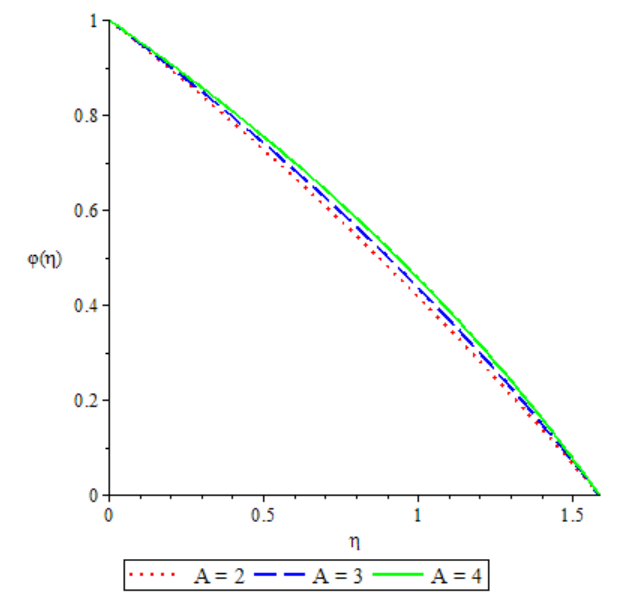

Figure 17. Concentration profiles for $\operatorname{Pr}=0.72, S c=0.24, E c=$ $0.5, D a=0.1, \varsigma=0.5$ and $n=B i=\delta=H a=F_{w}=0.1$

\section{Conclusions}

The effects of various embedded flow parameters on the skin friction, heat and mass transfer rates, dimensionless velocity, temperature and pollutant concentration are summarized as follows:

- The skin friction coefficient increases as the values of magnetic field, permeability and unsteadiness parameters are intensified but decreases in values as more fluid is injected into the flow system with an increasing slip parameter.

- The dimensionless plate temperature diminishes in values as the Biot number and slip parameter increase.

- The heat transfer rate increases as all flow parameters increase with an exception of the unsteadiness parameter perpetuating opposite influence.

- The Schmidt number, external pollutant source and slip parameters all have enhancing influence on the rate at which fluid mass is transferred from the plate surface to the far field stream while the Hartman number, fluid injection, unsteadiness and permeability parameters divulge its diminution.

- The dimensionless velocity appreciates in values as the

flow unsteadiness and fluid withdrawal parameters increase but dampens with an increase in the Hartman number.

- pollutant concentration increases with unsteadiness but dampens with an increase in the Hartman number.

- The fluid accelerates as the slip parameter is enhanced while the thermal boundary layer thickness decreases with an increase in fluid withdrawal parameter.

- The dimensionless wall temperature appreciates in values as the unsteadiness and permeability parameters, Prandtl, Eckert and Hartman numbers increase.

- The dimensionless fluid temperature rises when fluid withdrawal parameter and Biot number intensify.

- The pollutant concentration decreases as the fluid withdrawal and the local pollutant external source variation parameters increase but increases as the Schmidt number and the local pollutant external source parameter intensify.

- The pollutant concentration increases concurrently as the level of external pollutant infiltrating the flow rises higher

\section{Acknowledgments}

A. A expresses his profound gratitude to the Department of Mathematics, University of Lagos for support in providing us full facilities to carry out this research work.

\section{Nomenclature}

$\mathrm{x}, \mathrm{y}$

$\mathrm{u}, \mathrm{v}$

$\mathrm{v}_{w}, \mathcal{U}_{e}$

$T, T_{\infty}$

$C, C_{\infty}$

$T_{w}, C_{w}$

$p, \rho$

$\mathrm{a}, \mathrm{b}$

$\mathrm{Ha}, \mathrm{Pr}$

Ec, Sc

FW, A

$\mathrm{Q}, \mathrm{S}(\mathrm{C})$ :

$\mathrm{Bi}, \mathrm{Da}$

$\mathrm{B}_{0}, \mathrm{Dm}$

$\mathrm{k}, \mathrm{h}_{\mathrm{f}}$

$K_{P}, C_{P}$

$\gamma, \alpha$

$\beta, \delta$

$\varsigma, n$ coordinate axes along and normal to the plate velocity components along the $\mathrm{x}$ - and $\mathrm{y}$-axes wall mass flux and free stream velocities temperatures within and outside boundary layer pollutant concentrations within and outside boundary layer wall temperature and concentration fluid pressure and density real constants with respective units of reciprocal of time an concentration Hartman and Prandtl numbers Eckert and Schmidt numbers suction and unsteadiness parameters pollutant source strength and external pollutant concentration

Biot and Darcy numbers uniform magnetic field normal to the plate and mass diffusivity coefficient of pollutant Concentration thermal conductivity of the fluid and convective heat transfer coefficient permeability of the porous medium and specific heat of the fluid at constant pressure kinematic viscosity and thermal diffusivity of the fluid slip length and slip parameter pollutant external source and pollutant external source variation parameters 


$\begin{array}{ll}\psi, f & \begin{array}{l}\text { dimensional and dimensionless stream } \\ \text { functions }\end{array} \\ \theta, \phi & \begin{array}{l}\text { dimensionless temperature and concentration } \\ \text { fields. } \\ \text { electrical conductivity of the fluid and } \\ \text { similarity variable. }\end{array} \\ \end{array}$

\section{References}

[1] O. D. Makinde and T. Chinyoka, "Analysis of nonlinear dispersion of a pollutant ejected by an external source into a channel flow," Math. Prob. in Engng, vol. 2010, Article ID 827363, 17 pages, 2010.

[2] O. D. Makinde, R.J. Moitsheki, and B. A. Tau, "Similarity reductions of equations for river pollution," Appl. Math. Comput. vol.188, pp. 1267-1273, 2007.

[3] R.J. Moitsheki and O.D. Makinde, "Symmetry reductions and solutions for pollutant diffusion in a cylindrical system," Nonlinear Anal. RWA, vol. 10, pp. 3420-3427, 2009.

[4] R.J. Moitsheki and O.D Makinde, "Computational modelling and similarity reduction of equations for transient fluid flow and heat transfer with variable properties," Adv. in Mech. Engng, vol. 2013, Article ID 983962, 8 pages , 2013.

[5] T. Chinyoka and O.D. Makinde, “ Transient analysis of pollutant dispersion in a cylindrical pipe with a nonlinear waste discharge concentration,"Computers and Mathematics with Applications, vol. 60, pp. 642-652, 2010.

[6] K. Lakshminarayanachari, C.M. Suresha, M.S. Prasad and C. Pandurangappa, "A two dimensional numerical model of primary pollutant emitted from an urban area source with wet deposition and mesoscale wind," Int. J. Sci. Env. and Tech. vol. 2 , no 1 , pp: $60-79,2013$.

[7] M. Shekhu and C. Sulochana. "Time dependent mathematical model of air pollutants emitted from time-dependent elevated line source into a stable atmospheric boundary layer," J. Chem. Engng. and Mat. Sci., vol. 4, issue 8, pp. 103-115, 2013.

[8] H.I.Andersson, J.B. Aarseth and B.S. Dandapat, "Heat transfer in a liquid film on an unsteady stretching surface," Int. J. Heat and Mass Trans., vol. 43, no. 1, pp. 69-74, 2000.

[9] M.S. Abel, N. Mahesha and J. Tawade, "Heat transfer in a liquid film over an unsteady stretching surface with viscous dissipation in presence of external magnetic field," Appl.
Mathematical Modelling, vol.33, pp. 3430-3441, 2009.

[10] S. Mukhopadhyay, "Heat transfer in a moving fluid over a moving nonisothermal flat surface," Chin. Phys. Lett., vol. 8, no.12, ID124706, 2011.

[11] D.A. Nield and A. Bejan, Convection in Porous Media, $3^{\text {rd }}$ Ed., Springer Science + Business Media, Inc., N. York, 2006.

[12] D.B. Ingham and I. Pop, Transport Phenomena in Porous Media, Elsevier, Oxford, UK, 2005.

[13] I. Pop and D.B. Ingham, Convective Heat Transfer, Pergamon, Amsterdam, The Netherland, 2001.

[14] A. Ishak, N.A Yacob and N. Bachok,. "Radiation effects on the thermal boundary layer flow over a moving plate with convective boundary condition," Meccanica doi:10.1007/s11012-010-9338-4, 2010.

[15] P.O. Olanrewaju, F.I. Alao, A. Adeniyan, S.A. Bishop, "Double-diffusive convection from a permeable vertical surface under convective boundary condition in the presence of heat generation and thermal radiation," Nonlinear Sci. Lett. A., vol. 4, No.3 pp. 76-90, 2013.

[16] A. Adeniyan and J.A. Adigun, "Effects of chemical reaction on stagnation point mhd flow over a vertical plane with convective boundary conditions in the presence of a transverse uniform magnetic field," The Inter. J. Engng and Sci. (IJES), vol. 2 issue 4 pp.14-18, 2013.

[17] O.D. Makinde, "Computational modelling of mhd unsteady flow and heat transfer toward a flat plate with Navier slip and Newtonian heating," Braz. J. Chem. Engng, vol. 29, No. 01, pp. 159 - 166, 2012.

[18] K. Bhattacharyya, S. Mukhopadhya, G.C Layek, "Similarity solution of mixed convection boundary layer slip flow over a vertical plate," Ain Shams J.(Mech. Engng), vol. 4, pp.299305. 2013.

[19] S.Y. Ibrahim and O.D. Makinde, "Radiation effect on chemically reacting magnetohydrodynamics (mhd) boundary layer flow of heat and mass transfer through a porous vertical flat plate, Int. J. of Phy. Sci., vol. 6, issue 6, pp.1508-1516, 2011

[20] P. Dulal and P.S. Hiremath, “ Computational modeling of heat transfer over an unsteady stretching surface embedded in a porous medium," Meccanica..Vol. 45, issue 3, pp. 415-524, 2009. 